\title{
Short Fibrils Constitute the Major Species of Seed-Competent Tau in the Brains of Mice Transgenic for Human P301S Tau
}

\author{
Samuel J. Jackson, ${ }^{1 \star}$ Caroline Kerridge,${ }^{1 \star}$ Jane Cooper, ${ }^{1}$ Annalisa Cavallini, ${ }^{1}$ Benjamin Falcon, ${ }^{2}$ Claire V. Cella, ${ }^{1}$ \\ Alessia Landi, ${ }^{1}$ Philip G. Szekeres, ${ }^{1}$ Tracey K. Murray, ${ }^{1}$ Zeshan Ahmed, ${ }^{1}$ Michel Goedert, ${ }^{2}$ Michael Hutton, ${ }^{1}$ \\ Michael J. O'Neill, ${ }^{1}$ and Suchira Bose ${ }^{1}$ \\ ${ }^{1}$ Eli Lilly and Company Limited, Lilly Research Centre, Erl Wood Manor, Windlesham, Surrey GU20 6PH, United Kingdom, and ${ }^{2}$ MRC Laboratory of \\ Molecular Biology, Cambridge CB2 0QH, United Kingdom
}

The interneuronal propagation of aggregated tau is believed to play an important role in the pathogenesis of human tauopathies. It requires the uptake of seed-competent tau into cells, seeding of soluble tau in recipient neurons and release of seeded tau into the extracellular space to complete the cycle. At present, it is not known which tau species are seed-competent. Here, we have dissected the molecular characteristics of seed-competent tau species from the TgP301S tau mouse model using various biochemical techniques and assessed their seeding ability in cell and animal models. We found that sucrose gradient fractions from brain lysates seeded cellular tau aggregation only when large ( $>10$ mer) aggregated, hyperphosphorylated (AT8- and AT100-positive) and nitrated tau was present. In contrast, there was no detectable seeding by fractions containing small, oligomeric $(<6 \mathrm{mer})$ tau. Immunodepletion of the large aggregated AT8-positive tau strongly reduced seeding; moreover, fractions containing these species initiated the formation and spreading of filamentous tau pathology in vivo, whereas fractions containing tau monomers and small oligomeric assemblies did not. By electron microscopy, seed-competent sucrose gradient fractions contained aggregated tau species ranging from ring-like structures to small filaments. Together, these findings indicate that a range of filamentous tau aggregates are the major species that underlie the spreading of tau pathology in the P301S transgenic model.

Key words: aggregation; propagation; tau; tauopathy

\section{Significance Statement}

The spread of tau pathology from neuron to neuron is postulated to account for, or at least to contribute to, the overall propagation of tau pathology during the development of human tauopathies including Alzheimer's disease. It is therefore important to characterize the native tau species responsible for this process of seeding and pathology spreading. Here, we use several biochemical techniques to dissect the molecular characteristics of native tau protein conformers from TgP301S tau mice and show that seed-competent tau species comprise small fibrils capable of seeding tau pathology in cell and animal models. Characterization of seed-competent tau gives insight into disease mechanisms and therapeutic interventions.

\section{Introduction}

Microtubule-associated protein tau is enriched in nerve cell axons, where it is believed to stabilize microtubules. Native tau is converted into insoluble, filamentous, and hyperphosphorylated

\footnotetext{
Received Sept. 23, 2015; revised Nov. 17, 2015; accepted Nov. 27, 2015

Author contributions: S.J.J., P.G.S., M.J.O., and S.B. designed research; S.J.J., C.K., J.C., A.C., B.F., C.V.C., A.L., T.K.M., and Z.A. performed research; C.K., A.C., C.V.C., and Z.A. analyzed data; C.K., M.G., M.H., and S.B. wrote the paper.

We thank Dr. Ron DeMattos for his technical guidance with the nondenaturing gradient gel electrophoresis experiments, Alice Fisher for assistance with tissue sectioning, and Mark Ward for assistance with processing the tissue sections for the in vivo experiments.

The authors declare no competing financial interests.
}

inclusions in a number of human neurodegenerative diseases, collectively known as tauopathies (Spillantini and Goedert, 2013). During the course of Alzheimer's disease (AD), tau pa-

*S.J.J. and C.K. contributed equally to this work.

This article is freely available online through the J Neurosci Author Open Choice option.

Correspondence should be addressed to Suchira Bose, Eli Lilly and Company Limited, Lilly Research Centre, Erl Wood Manor, Sunninghill Road, Windlesham, Surrey GU20 6PH, UK. E-mail: bose_suchira@lilly.com.

S.J. Jackson's present address: NC3Rs, Gibbs Building, 215 Euston Road, London NW1 2BE, UK.

DOl:10.1523/JNEUROSCI.3542-15.2016

Copyright $\odot 2016$ Jackson, Kerridge et al.

This is an Open Access article distributed under the terms of the Creative Commons Attribution License Creative Commons Attribution 4.0 International, which permits unrestricted use, distribution and reproduction in any medium provided that the original work is properly attributed. 
thology appears to spread in a stereotypical spatial and temporal pattern, albeit with significant interpatient variability (Braak and Braak, 1991). The interneuronal transmission and spread have been demonstrated in several mouse models of tauopathy (Clavaguera et al., 2009; de Calignon et al., 2012; Ahmed et al., 2014; Liu et al., 2012). In one such model, the injection of brain extracts from TgP301S tau mice (with tau inclusions) into Alz17 mice (lacking tau inclusions), induced the slow assembly of endogenous tau into filaments, followed by its spread to anatomically connected brain regions (Clavaguera et al., 2009). Whereas detergent-insoluble brain fractions containing aggregated tau could seed pathology, soluble fractions were not (Clavaguera et al., 2009). Filamentous recombinant tau infused into brains of presymptomatic TgPS19 or TgP301S tau mice also converted soluble tau into hyperphosphorylated aggregates (Clavaguera et al., 2013a; Iba et al., 2013). We have recently developed a robust and rapid model of intraneuronal tau pathology propagation by infusing brain extracts from symptomatic TgP301S tau mice into the hippocampus of young, presymptomatic transgenic mice, resulting in the formation of filamentous tau pathology, which spread to the contralateral hippocampus and other synaptically connected brain regions (Ahmed et al., 2014). Similarly, the injection of brain extracts from human tauopathy patients into mice transgenic for human tau led to the formation of tau fibrils and the spreading of pathology (Clavaguera et al., 2013b; Sanders et al., 2014). Moreover, some of the characteristics of human tauopathies were preserved following injection, consistent with the existence of prion-like tau strains (Goedert, 2015).

Using mammalian and neuronal cell-based models, several studies have demonstrated the intercellular transfer of tau inclusions in vitro (Frost et al., 2009; Guo and Lee, 2011; Kfoury et al., 2012; Santa-Maria et al., 2012; Wu et al., 2013). Synthetic tau filaments made from recombinant tau or tau filaments extracted from $\mathrm{AD}$ brain were taken up by cells and induced the aggregation of cytoplasmic tau. Endocytosis at axonal and dendritic terminals with subsequent anterograde and retrograde transport of oligomeric tau forms has been demonstrated in primary neurons (Wu et al., 2013). This internalization of aggregated tau has been shown to depend on the presence of cell surface heparan sulfate proteoglycans (Holmes et al., 2013). To probe the mechanisms of tau pathology spreading further, we established a seeded tau aggregation cell-based assay using HEK 293T cells overexpressing 1N4R tau with the P301S mutation (Falcon et al., 2015). In this model, we showed that conformational differences might account for the superior seeding efficiency exhibited by tau aggregates extracted from the brains of TgP301S mice compared with recombinant $\mathrm{P} 301 \mathrm{~S}$ tau aggregates.

However, despite this recent progress, the mechanisms that underlie the spreading of filamentous tau pathology in human disease remain poorly understood and the characteristics of the tau species involved remain largely undefined. Although experimental models have demonstrated the spread of tau pathology (Clavaguera et al., 2009; Ahmed et al., 2014), the species that underlie the spreading of tau pathology were not defined. In the present study, we therefore determined the characteristics of tau from the brains of TgP301S tau mice with tau pathology in relation to its ability to seed formation of de novo aggregated tau in cell-based and in vivo models. Using sucrose gradient fractionation, nondenaturing gel electrophoresis, and immunodepletion, we show that seed-competent tau from brain lysates of symptomatic TgP301S mice consists predominantly of aggregated, highmolecular-weight species that include hyperphosphorylated and nitrated forms. The major seeds appear to be short filamentous structures with an average length of $179 \mathrm{~nm}$. We found no evidence of seed-competent small oligomeric tau assemblies.

\section{Materials and Methods}

Animals and cells. All animal procedures were performed in accordance with the Animals (Scientific Procedures) Act 1986 and were approved by the Eli Lilly Animal Welfare Board. HEK-293 T-Rex cells (Invitrogen) were stably transfected with 1N4R P301S tau under the control of a tetracycline promoter, as per the manufacturer's instructions (here called P301S-HEK). These cells were maintained at $37^{\circ} \mathrm{C} 5 \% \mathrm{CO}_{2}$ in DMEM, supplemented with tetracycline-free fetal bovine serum, and tau expression was induced by addition of $1 \mu \mathrm{g} / \mathrm{ml}$ tetracycline.

Antibodies. The following antibodies were kind gifts from Peter Davies (Albert Einstein College of Medicine, New York): total tau: DA9 (aa 102-140; Tremblay et al., 2010), TG5 (aa 220-240; Vincent et al., 1996); phosphorylated tau: PG5 (pS409; Jicha et al., 1999), PHF1 (pS396/404; Greenberg et al., 1992).

Phosphorylation-dependent anti-tau antibodies AT8 (pS202/pT205) and AT100 (pS212/pT214/pT217), as well as phosphorylation-independent antibody HT7 (aa 159-163), were purchased from Thermo (Pierce). An antibody specific for tau nitrated at Y29 (nY29) was purchased from Covance.

The generation and characterization of phosphorylation-independent antibodies BR133 (N-terminus), BR135 (repeat region), and BR134 (Cterminus) were previously described (Goedert et al., 1989).

Preparation of brain lysates and brainstem extracts from TgP301S tau mice. Mice transgenic for human P301S tau were euthanized by cervical dislocation and decapitation. Brains from presymptomatic (4.4 weeks) and symptomatic (24.4 weeks) TgP301S tau mice were homogenized in PBS plus complete protease inhibitor cocktail (Roche). Homogenates were pooled and spun at $13,000 \times g$ for $10 \mathrm{~min}$ at $4^{\circ} \mathrm{C}$. The supernatants were stored in aliquots at $-80^{\circ} \mathrm{C}$ until use. Symptomatic TgP301S mice were defined as animals that developed a neurological phenotype dominated by a severe parapesis (Allen et al., 2002).

Brainstem extracts were prepared to serve as a positive control for in vivo infusion studies (Ahmed et al., 2014). Brainstems from symptomatic TgP301S tau mice were rapidly dissected and snap-frozen. Brainstems from 5 mice were combined and homogenized via pulsed sonication in $10 \%(\mathrm{w} / \mathrm{v})$ sterile Dulbecco's PBS (DPBS) with a complete protease inhibitor cocktail, briefly sonicated and centrifuged for $5 \mathrm{~min}$ at $3000 \times \mathrm{g}$ at $4^{\circ} \mathrm{C}$. Supernatants were pooled, aliquoted and stored at $-80^{\circ} \mathrm{C}$. Wildtype brainstem extracts ( $\left.{ }^{\mathrm{WT}} \mathrm{BE}\right)$ were prepared from $\mathrm{C} 57 \mathrm{BL} / 6 \mathrm{~J}$ mice.

Sucrose gradient centrifugation. Ten, 20, 30, 40, and 50\% sucrose were prepared in water and layered onto a $12 \mathrm{ml}$ centrifuge tube in decreasing density. TgP301S tau brain lysate $(60 \mathrm{mg}$ protein in $4 \mathrm{ml}$ ) was added on top of the gradient and the tube spun at 250,000 $\times \mathrm{g}$ in a Sorvall Th641 swing-out rotor for $4 \mathrm{~h}$. Fractions were collected and stored at $-80^{\circ} \mathrm{C}$ until analysis. The PBS fraction remaining in the top of the sucrose gradient following centrifugation was called "Top." The pellet that formed at the bottom of the tube, was dislodged, disrupted by probe sonication into PBS, and stored at $-80^{\circ} \mathrm{C}$, unless otherwise stated. For analysis of Sarkosyl solubility, an aliquot of each fraction was incubated with $1 \%$ final volume of Sarkosyl for $1 \mathrm{~h}$ at room temperature, while shaking, before a $30 \mathrm{~min}$ centrifugation at $100,000 \times g$. The pellet contained Sarkosyl-insoluble tau.

Immunodepletion. Activated sepharose 4 Fast Flow (GE Healthcare) was conjugated to the antibody of choice at $5 \mathrm{mg} / \mathrm{ml}$, as per manufacturer's instructions, and stored in PBS containing $0.01 \%$ sodium azide (Sigma-Aldrich). Following washing, $250 \mu \mathrm{l}$ beads were incubated with 5 $\mathrm{mg}$ TgP301S tau brain lysate and rotated overnight at $4^{\circ} \mathrm{C}$. Sepharose was removed through centrifugation at $1000 \times g$ and depleted lysates stored at $-80^{\circ} \mathrm{C}$ until further analysis.

Seeding in a cell-based assay. HEK293T cells inducibly expressing human 1N4R P301S tau were used (Falcon et al., 2015). P301S-HEK cells were induced to express tau and subsequently incubated with inoculum, which consisted of either sucrose gradient fractions or immunodepleted lysates diluted in OptiMem (Life Technologies). After $3 \mathrm{~h}$, the inoculum was removed and replaced with fresh complete medium (DMEM plus $10 \%$ fetal calf serum). Cells were then grown for $3 \mathrm{~d}$. They were harvested 
and homogenized in sodium-phosphate buffer supplemented with protease (Roche) and phosphatase (Sigma-Aldrich) inhibitors. Fractionation of cell homogenates into insoluble and soluble proteins was achieved by a $1 \mathrm{~h}$ centrifugation at $100,000 \times g$. Total and hyperphosphorylated tau were measured in the pellet (insoluble) fraction by Western blotting, AlphaScreen and/or ELISA.

SDS-PAGE and Western blotting. Samples were boiled in sample buffer containing 2-mercaptoethanol, loaded onto Novex $8 \%$ Bis-Tris gels (Life Technologies) and run at $150 \mathrm{~V}$. Proteins were transferred onto nitrocellulose (GE Healthcare) in a semi-dry transfer tank, blocked with 5\% fat-free milk (Marvel) and immunoblotted using AT8, DA9, HT7, AT100, nY29, PHF1, BR133, BR134, or BR135. After secondary antibody incubation, bands were visualized using chemiluminescent substrate, and gels imaged and quantified using the ImageQuant LAS 4000 (GE Healthcare).

AlphaScreen. Levels of total (DA9) and phosphorylated (AT8) tau were quantified using AlphaScreen (Cavallini et al., 2013). Sample dilutions were incubated overnight at $4^{\circ} \mathrm{C}$ with antibody-bound Alphascreen acceptor beads (PerkinElmer) and antibody pair in 0.1\% Casein in PBS. Following binding of the sample to the antibody pair (biotinylated antibody and acceptor-bead bound antibody) overnight, AlphaScreen donor beads (PerkinElmer) were added to the appropriate concentration and incubated at room temperature for $4 \mathrm{~h}$ before reading the plate on an Envision plate reader (PerkinElmer).

Nondenaturing gradient gel electrophoresis. Large format 4-22\% acrylamide gels were poured using an SG100 gradient pourer (GE Healthcare) and pre-run in a Hoeffer 600SE electrophoresis tank (DeMattos et al., 2001). Protein normalized sucrose fractions $(5 \mu \mathrm{g})$ were loaded in nondenaturing sample buffer and run for $20 \mathrm{~h}$ at $4^{\circ} \mathrm{C}$. High Molecular Weight Native Marker Kit (GE Healthcare) and NativeMark (Invitrogen) were used. Proteins were transferred onto a polyvinylidene fluoride membrane and immunoblotted as described above.

Electron microscopy. Fractions were placed on 400 meshed Formvar/ carbon film-coated copper grids (Sigma-Aldrich) for $3 \mathrm{~min}$, blocked with PBS $+0.1 \%$ gelatin for 10 min and incubated with anti-tau antibody HT7 (1:50) or AT8 (1:100) for $1 \mathrm{~h}$. Grids were then washed and incubated with secondary antibody/10 nm gold conjugate (Sigma-Aldrich, 1:20) for $1 \mathrm{~h}$, before being washed and stained with uranyl acetate. Images were taken on a Philips Spirit transmission electron microscope at a magnification of 30,000 .

Stereotaxic infusion in TgP301S tau mice. Stereotaxic infusions were performed as described previously (Ahmed et al., 2014). Briefly female TgP301S tau mice (aged 10 weeks) were anesthetized with isoflurane ( $1-3 \%$ in $\mathrm{O}_{2}, 0.5 \mathrm{~L} / \mathrm{min}$ ), following sedation with Domitor, $1 \mathrm{mg} / \mathrm{kg}$ s.c. (medetomidine $\mathrm{HCl}$ ). When deeply anesthetized, they were placed in a stereotaxic frame (Stoelting) with a heating blanket and a rectal probe. Anesthesia was maintained with isoflurane during surgery. The skull was exposed and cleaned and a hole drilled over the appropriate coordinates for infusion. A $25 \mu$ l Hamilton syringe was secured in an infusion pump (CMA100, CMA) and connected via FEP tubing to a 28 -gauge cannula (Plastics One). The cannula was inserted slowly and $2.5 \mu \mathrm{l}$ brainstem extract or sucrose gradient fraction was unilaterally infused into the left hippocampus (AP: $-2.5 \mathrm{~mm}$; LM: $-2.0 \mathrm{~mm}$; DV: $-1.8 \mathrm{~mm}$ ) at a rate of $1.25 \mu \mathrm{l} / \mathrm{min}$. The cannula was left in position for $3 \mathrm{~min}$ to allow absorption of the bolus before administration of a second infusion into the overlying cortex (AP: $-2.5 \mathrm{~mm}$; LM: $-2.0 \mathrm{~mm}$; DV: $-0.8 \mathrm{~mm}$ ) Following surgery, incisions were sutured and glued, sedation reversed using Antisedan ( $5 \mathrm{mg} / \mathrm{kg}$, s.c.) and postoperative analgesia administered (Rimadyl, $5 \mathrm{mg} / \mathrm{kg}$, s.c.) (Carprofen). Mice were weighed and examined daily for 2 weeks with access to rehydration sachets, food, and water ad libitum.

Analysis of tau propagation in TgP301S tau mice. At 20 weeks of age (10 weeks postinfusion), mice were terminally anesthetized and transcardially perfused with saline, followed by $10 \%$ buffered formalin. The brains were removed and stored in formalin before being processed for immunohistochemistry as described previously (Ahmed et al., 2014). Paraffinembedded sections at bregma -2.30 and -3.00 (AP) were selected to analyze induction of tau pathology in the hippocampus and spread to the mammillary nucleus. Following deparaffinization and rehydration of the tissue sections, antigen retrieval was performed using the Lab Vision PT module system (Thermo Scientific), where sections were heated to $100^{\circ} \mathrm{C}$ for $20 \mathrm{~min}$ in citrate buffer (TA-250-PM1X; Thermo Scientific). Slides were transferred to a Lab Vision Autostainer (Thermo Scientific) where the following incubations were performed: 10 min in $\mathrm{H}_{2} \mathrm{O}_{2}(0.3 \%), 30$ min in normal goat serum (1:20; Vector Laboratories), 60 min in PG5 (1:8000); 30 min in biotinylated goat anti-mouse IgG (1:200, BA9200; Vector Laboratories), $30 \mathrm{~min}$ avidin-biotin complex solution (PK-7100; Vector Laboratories), and $5 \mathrm{~min}$ in 3,3'-diaminobenzidine (SK-4105; Vector Laboratories). Apart from the last two steps, PBS with $0.05 \%$ Tween 20 (PBS-T) was used for diluting reagents and washes between steps. Sections were then counterstained with hematoxylin before dehydration and coverslipping. To quantify PG5-positive tau pathology, stained sections were digitized using the Scanscope AT slide scanner (Aperio) at $20 \times$ magnification. Imagescope software (v11.1.2.780, Aperio) was used to view the digitized tissue sections and delineate boundaries of the left (infused) hippocampus, right hippocampus, and mammillary nucleus. PG5 immunoreactivity was quantified using the positive pixel algorithm (Imagescope v11.1.2.780, Aperio) and expressed as a percentage of the total area. These analyses were performed in a blinded fashion.

Statistical analysis. All analyses were performed using the Prism Graphpad software package. One-way ANOVA with Dunnett's post hoc test was used. Correlation and linear regression were performed to determine relationships between input and output tau $(n=3)$.

\section{Results \\ Sucrose gradient fractionation of TgP301S tau mouse brain lysates}

To characterize seed-competent tau, whole-brain lysates from presymptomatic (4.4 weeks) and symptomatic (24.4 weeks) TgP301S tau mice were fractionated by sucrose gradient centrifugation (Maeda et al., 2006). By Western blotting with anti-tau antibodies HT7 and DA9, all layers were found to contain tau, with a lower level of total tau in the densest fractions (40 and 50\% sucrose; Fig. 1A, top two panels). Antibodies BR133 (Nterminus), BR134 (C-terminus), and BR135 (repeat region) demonstrated that full-length tau was present in each fraction (data not shown). AT8- and AT100-positive tau migrating at 64 $\mathrm{kDa}$, was enriched in the $30-50 \%$ fractions of brain lysates from symptomatic TgP301S tau mice (Fig. $1 A$, third and fourth panels) consistent with these species being derived from high-molecularweight, but still SDS-labile, aggregates as previously reported (Allen et al., 2002; Berger et al., 2007). In contrast, much lower levels of these $64 \mathrm{kDa}$ hyperphosphorylated species were observed in the $20 \%$ fraction and this tau species was essentially absent in the $10 \%$ and Top fractions. The hyperphosphorylated $64 \mathrm{kDa}$ tau was also absent from the brain lysates of young presymptomatic TgP301S tau mice (Fig. 1A, third and fourth panels) again consistent with this tau species being derived from large pathological aggregates. Similar molecular weight tau species are characteristic of the pathologic tau observed in brain extracts from human tauopathies (Spillantini and Goedert, 2013). Consistent with previous findings in this model (Allen et al., 2002), we also observed AT8-positive tau migrating at $55 \mathrm{kDa}$ in the lysates of both presymptomatic and symptomatic TgP301S tau mice (Fig. 1A, AT8 blot). This species was enriched in the lower density fractions (Top, 10\%, 20\%) of symptomatic TgP301S mice, consistent with this tau being derived from smaller assemblies or reflecting the presence of AT8-positive monomeric tau.

Tau from the $30-50 \%$ sucrose gradient fractions of symptomatic mice contained Sarkosyl-insoluble material which was AT8positive and migrated exclusively at $64 \mathrm{kDa}$ on denaturing gels (Fig. $1 B$ ). By contrast, the $10 \%$ fraction did not contain any AT8positive Sarkosyl-insoluble tau. The $20 \%$ fraction contained a 


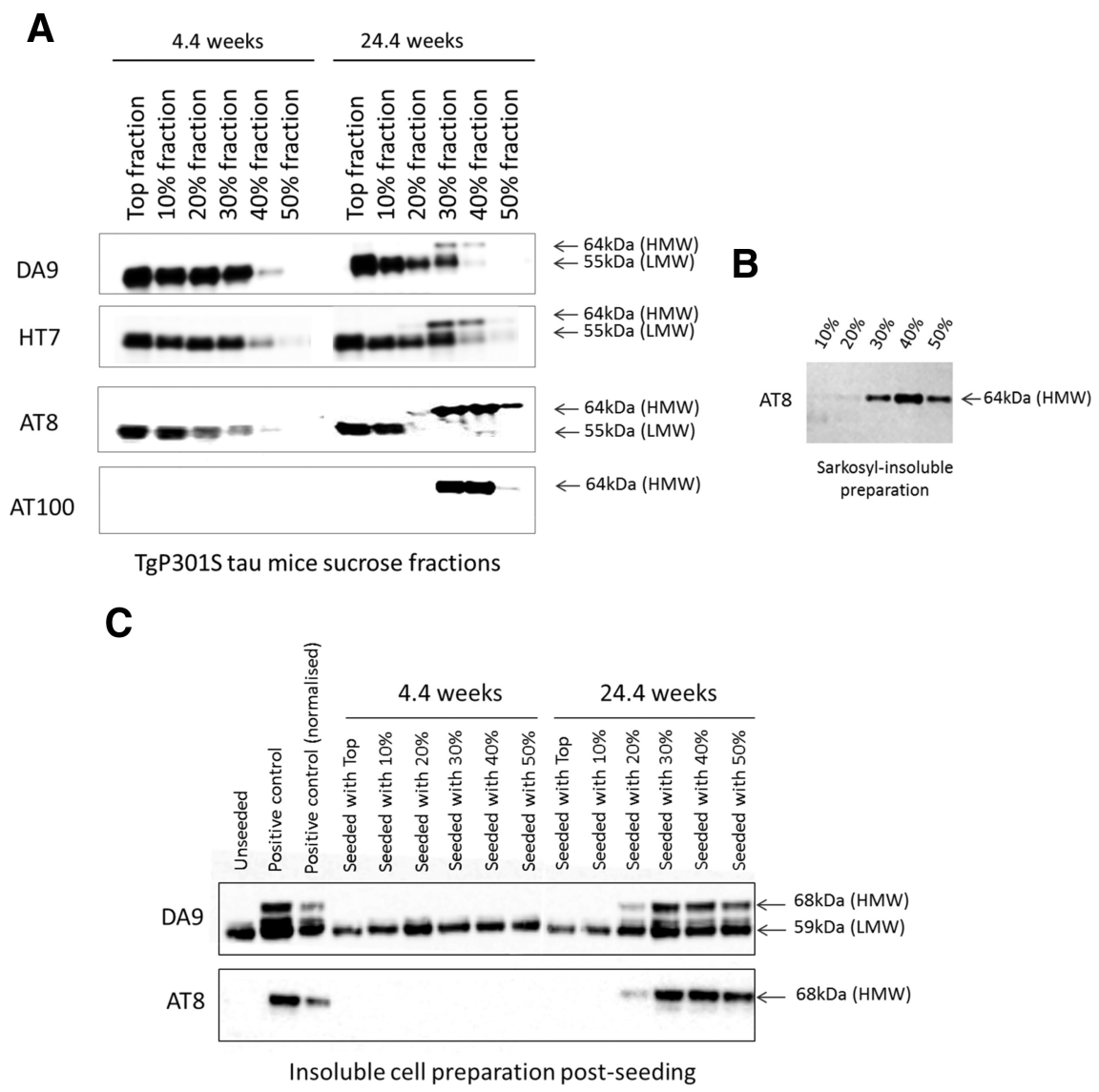

Figure 1. A, Sucrose gradient centrifugation of TgP301S tau mouse brain lysates to separate soluble and insoluble tau. Western blot with anti-tau antibodies DA9, HT7 (both phosphorylation-independent), AT8 (phosphorylation at pS202/pT205), and AT100 (phosphorylation at pS212/pT214/pT217) of symptomatic (24.4 weeks) and presymptomatic (4.4 weeks) total-brain lysate following sucrose gradient fractionation for $4 \mathrm{~h}$; gels loaded with $5 \mu \mathrm{g} /$ well of each fraction. Representative blots from 3 independent sucrose gradient fractionations are shown. Filamentous tau runs at $\sim 64 \mathrm{kDa}$ (high-molecular weight; $\mathrm{HMW}$ ). Nonfilamentous tau runs at $\sim 55 \mathrm{kDa}$ (low-molecular-weight; LMW). B, Representative Western blot of Sarkosyl-insoluble AT8-positive tau in sucrose gradient fractions of symptomatic TgP301S mouse brain. C, Seeding of tau aggregation with sucrose gradient fractions in a cell-based assay. Sucrose gradient fractions were used to seed aggregation of tau in HEK293T cells overexpressing 1N4R tau with the P3015 mutation. The pellet from a 100,000 $\times g$ spin of the seeded cells was analyzed by Western blotting using anti-tau antibodies AT8 and DA9. A representative blot is shown; similar results were obtained in three separate experiments. Filamentous tau runs at $\sim 68 \mathrm{kDa}$ (HMW). Nonfilamentous tau runs at $\sim 59 \mathrm{kDa}$ (LMW). Positive control was seeding with Sarkosyl extracted tau from symptomatic TgP3015 mice as described in Falcon et al. (2015) and the normalized positive control was seeding with Sarkosyl extracted tau from symptomatic TgP3015 mice, normalized for DA9 tau levels to those of the symptomatic sucrose gradient fractions. Seeding ability correlated with the presence of the $64 \mathrm{kDa}$, hyperphosphorylated tau band in symptomatic ( 24.4 weeks) mice. No seeding was observed upon addition of the sucrose gradient fractions from presymptomatic (4.4 weeks) mice.

mixture of Sarkosyl-soluble and Sarkosyl-insoluble tau. This demonstrates that hyperphosphorylated and aggregated highmolecular-weight (HMW) tau traveled to the denser sucrose fractions $(20-50 \%)$, whereas soluble tau remained in the less dense fractions (Top, $10-20 \%$ ).

\section{Tau aggregates positive with AT8 and AT100} are seed-competent

We next investigated the ability of the sucrose gradient fractions to seed the aggregation of 1N4R P301S tau in HEK293T cells (Falcon et al., 2015). The cells were induced to express 1N4R P301S tau and treated with sucrose gradient fractions for $3 \mathrm{~h}$, washed and incubated for a further $3 \mathrm{~d}$. They were then fractionated (in the absence of Sarkosyl) by high-speed centrifugation and insoluble tau detected by immunoblotting with DA9 and AT8 (Fig. 1C). Aggregated 1N4R tau was detected following treatment with the $30-50 \%$ sucrose fractions from symptomatic
TgP301S tau mice as shown by the formation of $68 \mathrm{kDa}, \mathrm{DA} 9-$ and AT8-positive tau (Fig. 1C). Low levels of tau seeding were also observed following treatment with the $20 \%$ sucrose gradient fraction, while tau species from the $10 \%$ sucrose gradient fraction were unable to seed tau aggregation. No seeding was observed with fractions from presymptomatic TgP301S tau mice (Fig. 1C).

We then normalized the relative levels of AT8 in the $30-50 \%$ sucrose fractions and used the normalized fractions to seed tau aggregation in the cell model. Quantification of the Western blot and analysis by Alphascreen demonstrated that the $40 \%$ fraction seeded the formation of significantly more aggregated $1 \mathrm{~N} 4 \mathrm{R}$ tau in the cell model than either the 30 or $50 \%$ fractions (Fig. $2 A, B$ ). This was despite the $30 \%$ sucrose fraction containing more total tau than the $40 \%$ fraction (Fig. $1 A$, DA9 and HT7 blots).

\section{Aggregated tau morphology in sucrose gradient fractions}

To evaluate the morphologies of tau aggregates, we examined the sucrose gradient fractions by immunoelectron microscopy with HT7 [Fig. 3A (presymptomatic mice), $B$ (symptomatic mice)].

The Top and $10 \%$ fractions from symptomatic TgP301S tau mice contained sparse immunogold labeling associated with amorphous structures. The $20 \%$ fraction contained a mixture of sparse and small, ring-like immunogold labeled tau-positive structures. The 30\% fraction contained globular, ring-like taupositive structures. The 40 and $50 \%$ fractions contained small, labeled filamentous structures similar to those seen in Sarkosyl-insoluble preparations (Falcon et al., 2015). They were significantly shorter in the $40 \%(179 \pm 12 \mathrm{~nm}, n=25)$ than in the $50 \%$ fraction $(250 \pm 15 \mathrm{~nm}$, $n=25)$. Sparse labeling was observed for all of the sucrose fractions from presymptomatic TgP301S tau mice (Fig. 3A).

\section{Nondenaturing gradient gel electrophoresis shows that seed- competent tau is composed of high-molecular-weight aggregates}

We characterized tau in the sucrose gradient fractions by nondenaturing gradient gel electrophoresis, to further understand the nature of seed-competent tau from the brains of TgP301S tau mice (Fig. 4A,B).

In symptomatic mice (Fig. 4B), DA9-positive tau was detected in all fractions, consistent with SDS-PAGE (Fig. 1A). In the Top, $10 \%$, and $20 \%$ fractions, DA9-positive tau comprised a range of molecular weights and produced a smear extending from $\sim 140$ $\mathrm{kDa}$ to $>1000 \mathrm{kDa}$ by comparison with molecular weight markers of defined hydrodynamic radii (DeMattos et al., 2001). Upon overexposure, monomeric tau could be faintly detected at $\sim 60$ 
A

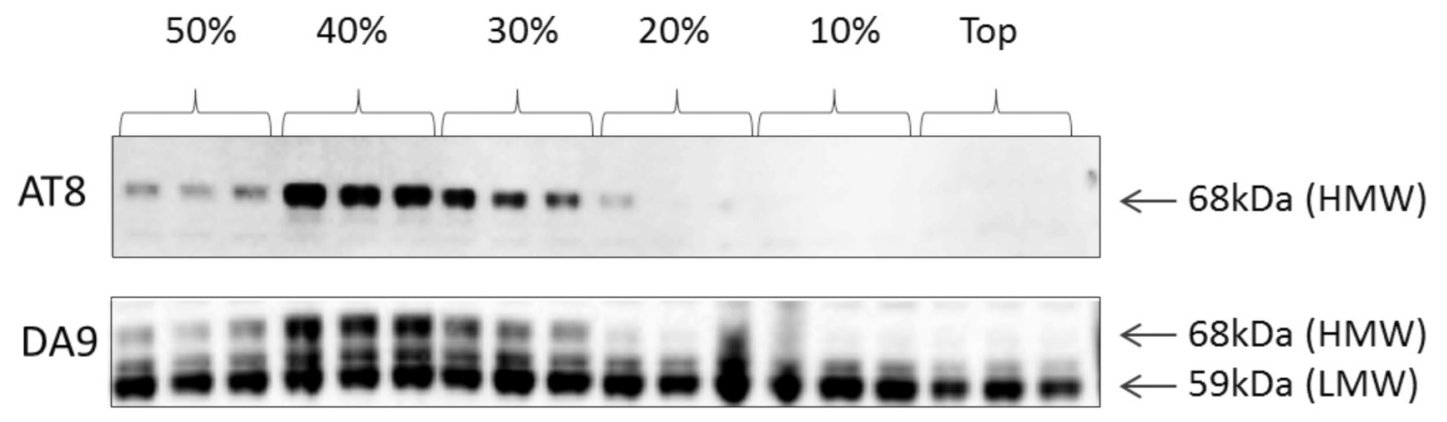

Insoluble cell preparation post-seeding

B

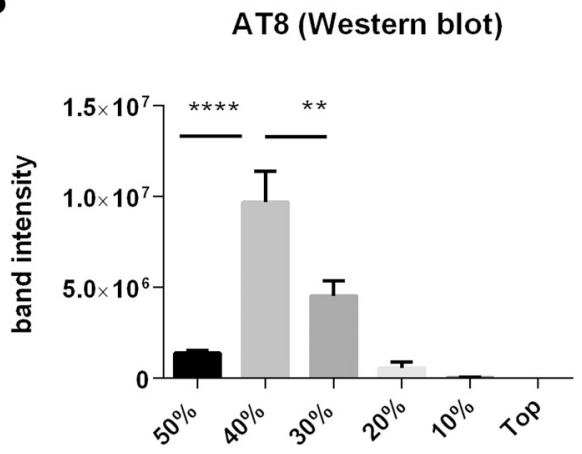

AT8 (AlphaScreen)

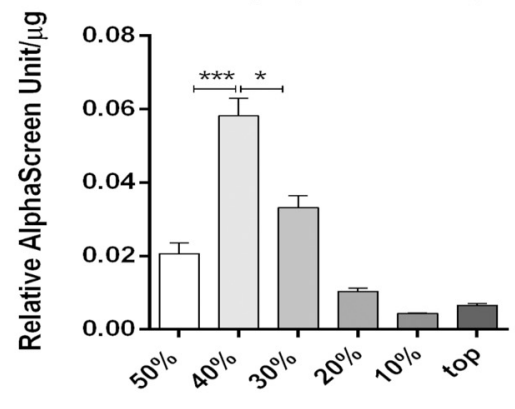

Figure 2. A, The AT8 normalized sucrose gradient fractions (30-50\% fractions) were tested for seeding efficiency in the cell-based assay. Western blots with AT8 and DA9 was used to detect seeding in the insoluble cell fraction following incubation with sucrose fractions. B, Quantification of the Western blot shown in A (left) and AlphaScreen analysis (right). The results are expressed as means $\pm \operatorname{SEM}(n=3) .{ }^{* *} p<0.01,{ }^{* * *} p<0.0001$ (one-way ANOVA + Dunnett's post hoc test relative to $40 \%$ fraction). The results confirmed that the $40 \%$ sucrose gradient fraction seeded significantly better than the 30 and $50 \%$ fractions.

$\mathrm{kDa}$ (data not shown). In contrast, in the 30,40 , and $50 \%$ fractions the majority of DA9-positive tau was large in size, as indicated by the fact that it remained in the wells.

In the Top, $10 \%$ and $20 \%$ fractions phosphorylated (AT8) and nitrated (nY29) tau (Fig. $4 B$ ), was detected that migrated at $\sim 400$ $\mathrm{kDa}$ by comparison with molecular weight markers of defined hydrodynamic radii (DeMattos et al., 2001). While size estimation on nondenaturing gels is difficult, this species would appear to represent a small phosphorylated and nitrated (low- $n$ ) tau oligomer, most likely a 6 mer. In the $30-50 \%$ and pellet fractions the majority of AT8-, PHF1-, and nY29-positive tau existed as large aggregates unable to enter the gel. These high-molecularweight species were estimated to be $>1240 \mathrm{kDa}$ ( $>10$ mer). Lower levels of these large aggregated phospho-tau species were also detected in the $20 \%$ fraction.

By comparing the levels of different tau species in the sucrose fractions with the seeding ability of each fraction it is clear that the extent of seeding (Fig. 1C) is related to the level of the large phosphorylated (AT8/PHF1) and nitrated (nY29) tau species that were abundant in the 30,40 , and $50 \%$ fractions, but absent from the Top and $10 \%$ fractions. In contrast, there was no evidence of pathological tau seeding by small oligomeric $(<10$ mer $)$ tau species in this analysis given that no seeding was observed with the Top and $10 \%$ fractions that appear to contain such species.

In presymptomatic mice, tau was mostly detected in the Top and 10\% fractions using the total tau antibody DA9 (Fig. $4 A)$. As in the symptomatic mice, DA9-positive tau in these low-density fractions comprised a wide range of molecular weights and produced a smear. AT8- and nY29-positive tau migrating at $\sim 400 \mathrm{kDa}$ in the Top and $10 \%$ fractions was also observed; however, this species was present at much lower levels than in symptomatic mice, indicating that it accumulated with ongoing tau pathogenesis. Importantly, high-molecular-weight tau that was positive with AT8 and nY29 was absent in presymptomatic mice and none of the sucrose fractions from presymptomatic mice were able to seed tau aggregation in our cell-based assay (Fig. 1C).

\section{Reduced seeding following immunodepletion of tau}

To further characterize the nature of seed-competent tau in the TgP301S transgenic model, we assessed the seeding abilities of brain lysates from symptomatic TgP301S tau mice following immunodepletion with a number of anti-tau antibodies (both phosphorylation-independent and phosphorylation-dependent). Immunodepletion was performed using DA9, AT8, PG5, PHF1, and total IgG (as a control). Brain lysates were analyzed by Western blotting and ELISA (data not shown) to quantify the extent of depletion of DA9- and AT8-positive species (Fig. 5A,B). Immunodepletion with DA9 removed DA9 reactivity and reduced the amount of 64 $\mathrm{kDa}$ AT8-positive tau (Fig. 5A,B). In contrast, immunodepletion with AT8, PG5, or PHF1 did not reduce total tau (DA9) levels but significantly reduced $64 \mathrm{kDa}$ aggregate-derived AT8-positive tau [AT8 $(-96 \%)>$ PHF1 $(-72 \%)>$ PG5 $(-70 \%)=$ DA9 $(-70 \%)$ relative to control IgG; Fig. $5 A, B]$. Depletion with IgG did not reduce DA9 or AT8 levels (Fig. 5A,B). 
A

4.4 weeks

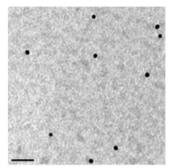

Top

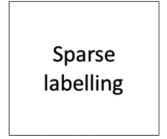

B

\section{4 weeks}
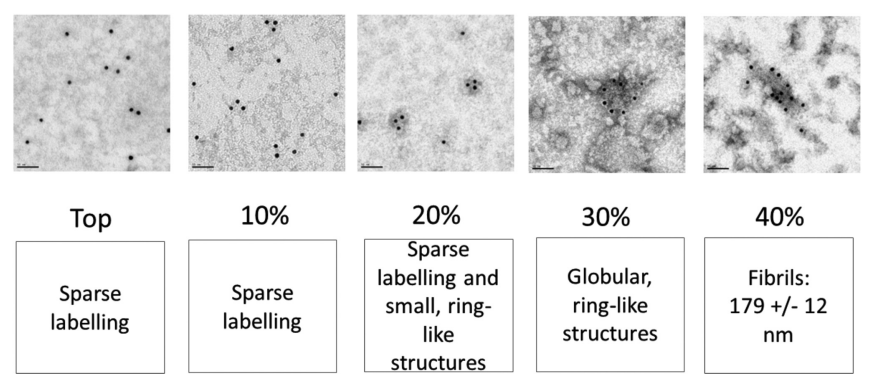

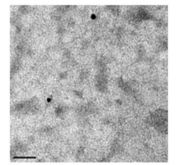

$40 \%$

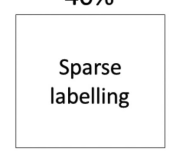

$50 \%$

Sparse

labelling
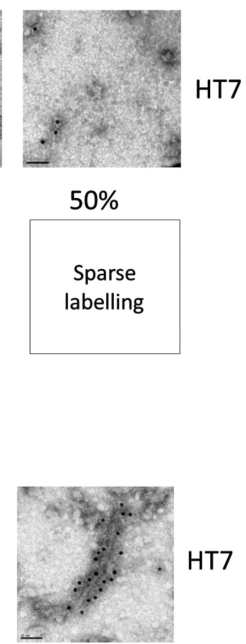

HT7
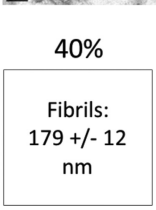

Figure 3. Immunoelectron microscopy of sucrose gradient fractions from the brains of presymptomatic (4.4 weeks) and symptomatic (24.4 weeks) TgP301S tau mice. Anti-tau antibody HT7 (phosphorylation-independent) was used to detect tau. $\boldsymbol{A}$, For presymptomatic mice, only sparse labeling was observed in the Top, $10-50 \%$ sucrose gradient fractions. $\boldsymbol{B}$, For symptomatic mice, tau filaments were present in in the $50 \%$ ( $250 \pm 15 \mathrm{~nm}, n=25)$ and $40 \%$ fractions $(179 \pm 12 \mathrm{~nm}, n=$ 25). The $30 \%$ fraction contained globular, ring-like tau-positive structures. The $20 \%$ fraction comprised a mixture of sparse labeling and small, ring-like tau-positive structures. The Top and $10 \%$ fraction showed only sparse tau labeling. A typical experiment is shown; similar results were obtained in three separate experiments. Scale bar, $50 \mathrm{~nm}$.

We then tested the seeding ability of the immunodepleted samples in HEK293T cells expressing 1N4R P301S tau. Immunodepletion with anti-tau antibodies, but not control IgG, reduced the seeding ability of TgP301S tau brain lysates (Fig. 5C,D). There was also a strong positive correlation $\left(R^{2}=0.85, p<\right.$ 0.0001 ) between the levels of $64 \mathrm{kDa}$ AT8-positive tau remaining in the brain lysates (AT8 input) and the levels of seeded aggregation observed in the cells, as measured by the levels of AT8positive insoluble tau following incubation with each immunodepleted brain lysate (AT8 output; Fig. 5E, left graph). No correlation was observed between the levels of total, DA9positive tau (comprising soluble and insoluble tau) in brain lysates (DA9 input and the levels of seeded aggregation AT8 output; Fig. 5E, right graph).

\section{High-molecular-weight tau aggregates are key mediators of tau pathology seeding and propagation in vivo}

Having established that seed-competent tau from symptomatic TgP301S tau mice comprises hyperphosphorylated, aggregated tau (ranging from ring-like structures to filaments of $250 \mathrm{~nm}$ ) based on our cell-based assay, we next determined whether similar tau species would support tau pathology seeding and propagation in vivo. To this end, we used an in vivo model of tau propagation in which we have previously shown robust induction of neuronal tau pathology and spreading to synaptically connected nuclei (Ahmed et al., 2014). The $10 \%$ sucrose gradient fraction (containing mainly $55 \mathrm{kDa}$ tau species; Fig. $1 A$, DA9 blot) and the $40 \%$ fraction (containing $64 \mathrm{kDa}$ AT8-positive filaments; Figs. $1 A$, AT8 blot, $4 B$ ), together with total brainstem lysate from symptomatic TgP301S tau mice, were stereotaxically infused into the left hippocampus and overlying cerebral cortex of presymptomatic TgP301S tau mice. After 10 weeks the mice were euthanized, their brains harvested, fixed in formalin and analyzed by immunohistochemistry (Fig. 6A). Total brainstem lysates and the $40 \%$ sucrose gradient fractions, but not the $10 \%$ fractions, showed a robust induction of tau pathology in the infused hippocampus; neurofibrillary pathology was also observed in synaptically connected regions, including the contralateral hippocampus, the mammillary nucleus, and the retrosplenial cortex (Fig. 6B, $C$, and data not shown).

Total brainstem lysates showed significantly more induction and spread of tau pathology than the $40 \%$ fractions (Fig. $6 B, C$ ). However, infusion of a total brainstem lysate preparation that was normalized to the same level of total tau (DA9) as the 10 and $40 \%$ sucrose gradient fractions demonstrated lower (nonsignificant) levels of tau induction and spread compared with the $40 \%$ fraction (Fig. 6 B, C). Thus, the $40 \%$ sucrose gradient fraction not only induced tau pathology in vivo, but also contained a higher concentration of seed-competent tau than the total brainstem lysate. These results are consistent with the hypothesis that tau aggregates, especially small tau filaments, which are the major tau species observed in the $40 \%$ sucrose fraction, are key mediators of seeding and propagation in vivo. Interestingly, neither the total brainstem lysate, nor the 10 or $40 \%$ sucrose fractions induced overt neuronal loss for up to 2.5 months after infusion into TgP301S tau mice (data not shown).

\section{Discussion}

Aggregated tau species can spread between anatomically connected neurons and template or seed the assembly of soluble tau into filamentous aggregates inside cells (Brettschneider et al., 2015; for review, see Goedert, 2015). The native tau species responsible for seeding are unknown. To start to bridge this gap, we performed extensive biochemical analysis of crude brain lysates from symptomatic TgP301S tau mice (Allen et al., 2002), that had previously been shown to be sufficient to support tau propagation, and used both a cell-based assay (Falcon et al., 2015) and an in vivo assay (Ahmed et al., 2014) to determine which tau species were seed-competent (capable of seeding tau aggregation and spreading). Previously, when brain lysates from symptomatic TgP301S tau mice were injected intracerebrally into mice transgenic for wild-type human tau, they induced aggregation of wildtype human tau and spreading of this pathology to connected brain regions (Clavaguera et al., 2009). Moreover, when infused either intracerebrally or intraperitoneally into young presymptomatic TgP301S tau mice, that lack significant forebrain tau pathology, brain lysates from symptomatic mice promoted the aggregation and spreading of P301S tau pathology (Clavaguera et al., 2013a; Ahmed et al., 2014).

To examine the conformers of native tau and their seeding ability, total brain lysate from TgP301S mice was separated by sucrose gradient fractionation (Maeda et al., 2006), which separates proteins according to size and density. Highly aggregated forms have a greater sedimentation coefficient and pellet 
A

\section{4 weeks}

DA9

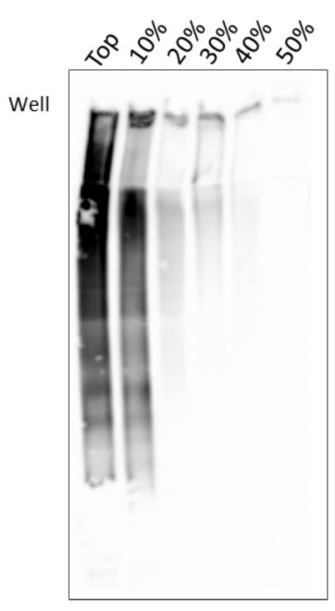

AT8

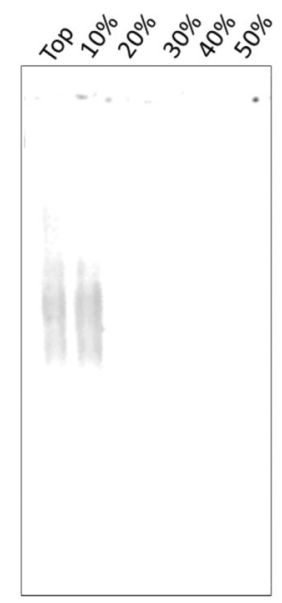

nY29

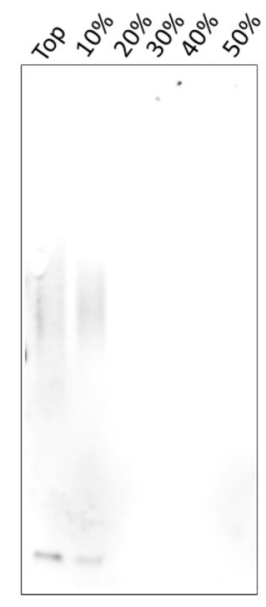

PHF1

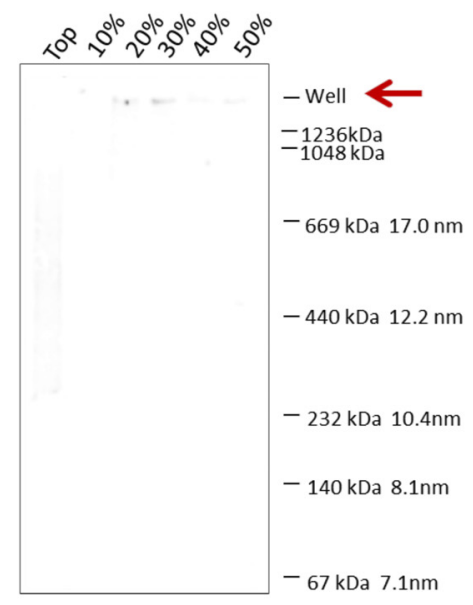

B

\section{4 weeks}

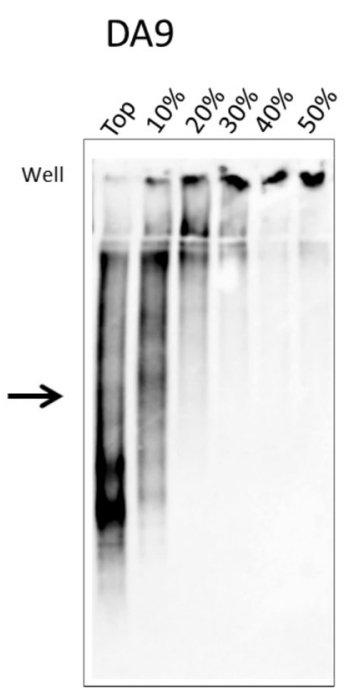

AT8

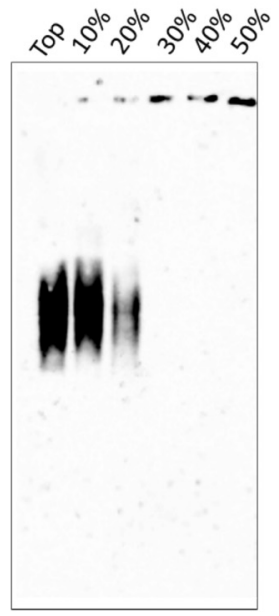

nY29

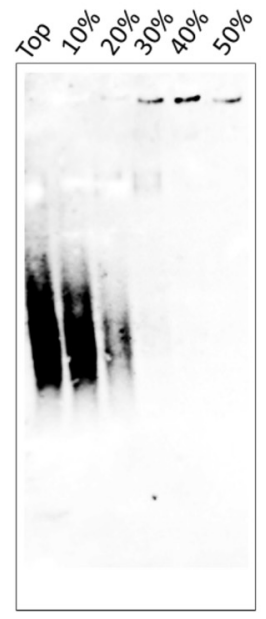

PHF1

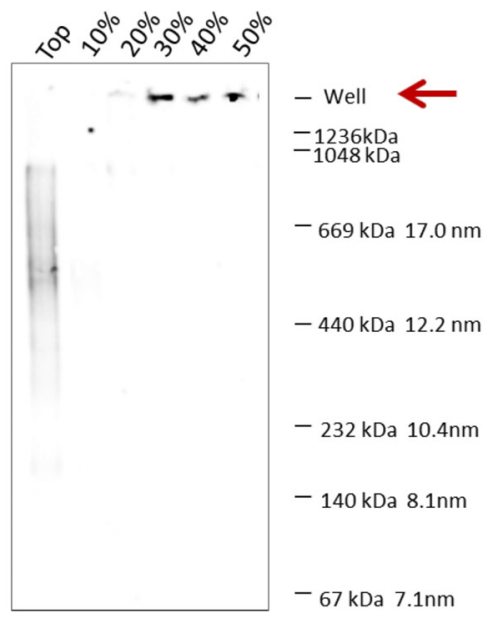

Figure 4. Nondenaturing gradient gel electrophoresis of sucrose gradient-fractionated TgP3015 tau mouse brain lysates. Four to 22\% nondenaturing gels of presymptomatic $(\boldsymbol{A})$ and symptomatic (B) TgP301S sucrose gradient fractions was performed ( $5 \mu \mathrm{g} /$ well of protein normalized fractions). Western blots with phosphorylation-independent anti-tau antibody DA9, phosphorylationdependent antibodies AT8 and PHF1, and an antibody specific for tau nitrated at Y29 (nY29). In the presymptomatic mice, the Top, 10\%, and 20\% fractions, tau species were recognized by the phosphorylation-independent pan tau antibody, DA9. Some of these tau species showed low-level reactivity with AT8 and nY29 antibodies but were PHF1-negative. In symptomatic mice, in the Top, $10 \%$, and $20 \%$ fractions a seeding-incompetent tau species of $\sim 400 \mathrm{kDa}$ was recognized by AT8 and $\mathrm{nY} 29$ (black arrow).In the $30-50 \%$ fractions, tau species were unable to migrate into the gel (HMW species; red arrow) and were recognized by all four antibodies. Representative blots from three independent experiments shown.

more rapidly than amorphous aggregates, small oligomers, and monomeric tau. The $40 \%$ sucrose fraction from the brains of TgP301S tau mice gave the most efficient seeding of aggregated tau in our cell model and robust initiation of pathology in vivo. This fraction contained insoluble high-molecular weight, aggregated tau that was phosphorylated (AT8) and nitrated (nY29). Electron microscopy further showed that the predominant tau assemblies in this fraction were short fibrils (average length of $179 \mathrm{~nm}$ ). Tau in the 20, 30, and 50\% fractions was also seed-competent in our cell model, albeit to a lesser ex- tent, consistent with the presence of some aggregated insoluble tau in these fractions that was detected by AT8 and nY29. In contrast, soluble tau species in the Top and 10\% sucrose gradient fractions were seed-incompetent both in our cellular assay (Top and $10 \%)$ and in vivo (10\%). Tau species in sucrose fractions from presymptomatic mice were also seed-incompetent, consistent with the absence of large aggregated insoluble tau. This was supported by immunodepletion experiments, which showed that a reduction in filamentous tau decreased the amount of seedcompetent tau. 
A

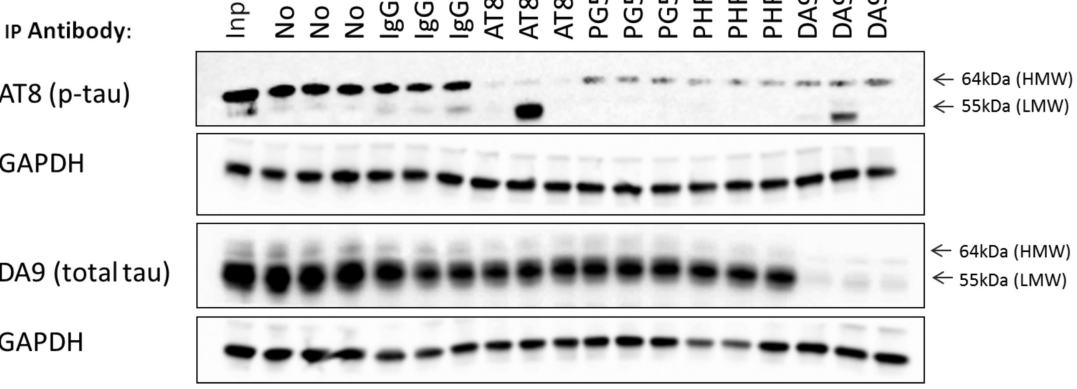

B
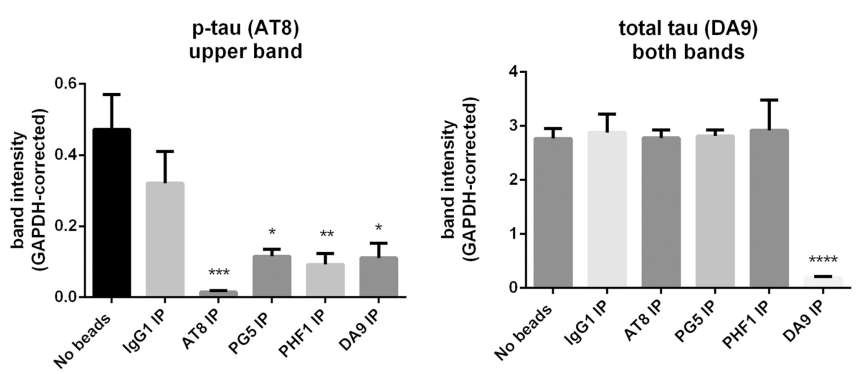

C

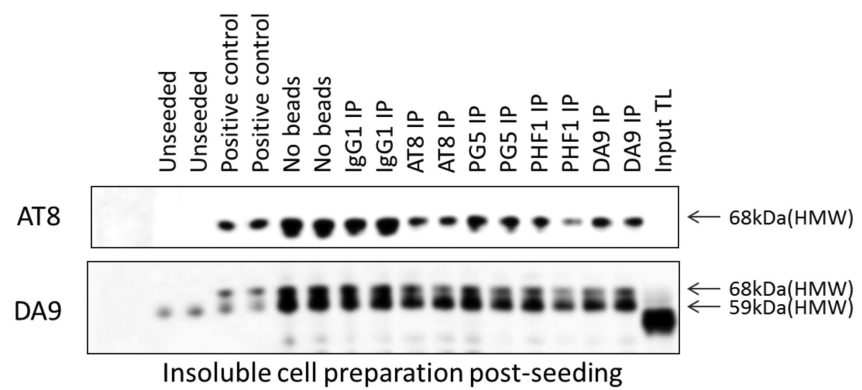

D
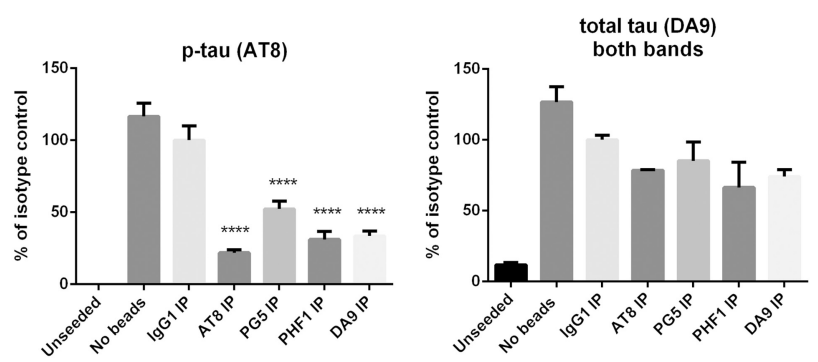

$\mathbf{E}$
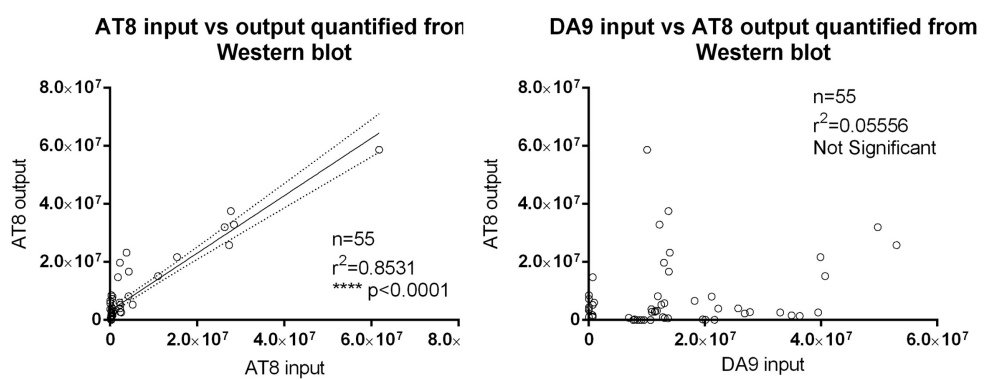

Figure 5. Seeding efficiency correlates with level of $64 \mathrm{kDa}$ hyperphosphorylated tau. $\boldsymbol{A}$, Western blot with AT8 or DA9 antibodies and ( $\boldsymbol{B}$ ) quantification of brain lysates from symptomatic TgP3015 tau mice (24.4 weeks) following immunodepletion with phosphorylation-independent anti-tau antibody DA9 or phosphorylation-dependent antibodies AT8, PG5, and PHF1. IgG1 served as the control. A representative blot from three separate experiments is shown, each with three replicates per condition. GAPDH was used as the loading control. Immunodepletion reduced levels of the $64 \mathrm{kDa}$ (HMW) AT8-positive band by all antibodies to varying degrees (AT8 > PHF1 > PG5 = DA9); only DA9 significantly reduced the $55 \mathrm{kDa}$ (low-molecular-weight; LMW) tau species. The quantification results are the mean $\pm \operatorname{SEM}(n=6) ; 64 \mathrm{kDa}$ band quantified for AT8 blot, and the sum of 64 and $55 \mathrm{kDa}$
By native PAGE, several types of tau aggregates were observed upon fractionation of brain lysates from symptomatic TgP301S tau mice. DA9-positive tau resolved as a ladder ranging from $\sim 140$ to $>1000 \mathrm{kDa}$ in the low-density fractions (Top, $10 \%$, and $20 \%$ ), in contrast in the higher density fractions (30-50\%) highmolecular-weight tau $(>1240 \mathrm{kDa})$ was the predominant species. The latter species did not enter the gel and were DA9-, AT8-, PHF1-, and nY29-positive, consistent with them being Sarkosyl-insoluble aggregates comprising various morphologies. The AT8 and nY29 antibodies also detected apparent oligomeric species migrating as a smear $\sim 400 \mathrm{kDa}$ in the Top and $10 \%$, but not in the higher density fractions. The $20 \%$ fraction contained a mixture of the AT8- and nY29-positive $400 \mathrm{kDa}$ smear and high-molecular-weight $(>1240 \mathrm{kDa})$ tau, consistent with this fraction showing some seeding ability but less than the $30-50 \%$ fractions. Smearing may indicate that the tau molecules within the $400 \mathrm{kDa}$ oligomeric species have differing levels of post-translational modification, which would account for small size differences. Consistent with the native PAGE results, immunoelectron microscopy confirmed the presence of multiple tau species with varying morphologies in the sucrose fractions. In symptomatic TgP301S tau mice, the Top and 10\% fractions showed sparse labeling associated with amorphous structures. The $20 \%$ fraction showed a mixture of sparse and small, ring-like tau-positive structures. The $30 \%$ fraction showed globular, ring-like taupositive structures, whereas the 40 and $50 \%$ fractions contained tau filaments like those from Sarkosyl-insoluble tau preparations from transgenic mice and human tauopathy cases (Spillantini and Goedert, 2013). Tau filaments in the $50 \%$ fraction were significantly longer

$$
\leftarrow
$$

bands quantified for DA9. C, Western blot and quantification (D) with anti-tau antibodies DA9 and AT8 of the insoluble cell fraction, following seeding with immunodepleted samples. Positive control was Sarkosyl extracted tau from symptomatic TgP301S mice as described by Falcon et al. (2015). A representative blot from three separate experiments is shown. $\boldsymbol{D}$, Results are the means \pm SEM of three separate experiments; $68 \mathrm{kDa}$ band quantified for AT8 blot, and the sum of 68 and $59 \mathrm{kDa}$ bands quantified for DA9. Statistical analysis: one-way ANOVA with Dunnett's post hoc test. ${ }^{*} p<0.05,{ }^{* * * *} p<0.0001$, compared with lgG1.E, The levels of $64 \mathrm{kDa}$, AT8-positive tau in the brain lysate correlated with the seeding ability measured by Western blotting. No correlation was observed with DA9-positive tau as the input. Linear regression was performed to determine the relationships between input and output tau ( $r^{2}=0.8531, p<0.0001, n=55$ for AT8-positive tau). 

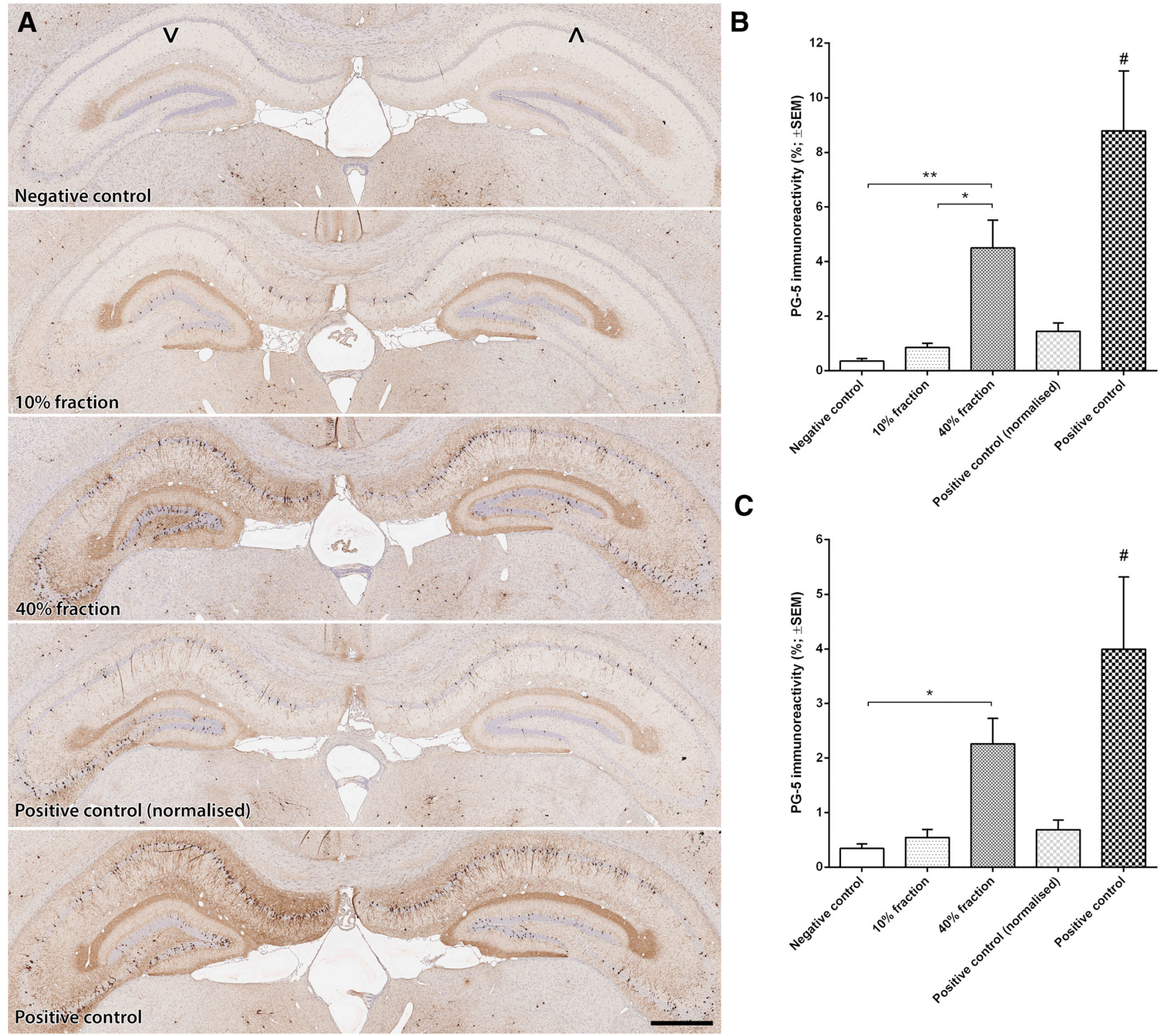

Figure 6. Tau-species in the $40 \%$ sucrose gradient fraction from the brains of symptomatic TgP301S tau mice (24.4 weeks) are key mediators of seeding and spreading. A, Unilateral infusion of brainstem lysate (positive control) from symptomatic TgP3015 mice into the hippocampus of asymptomatic TgP301S mice resulted in the robust accumulation and spread of PG-5-positive tau pathology in the infused $(\vee)$ and contralateral $(\wedge)$ hippocampus, respectively. Mice infused with brainstem lysate from wild-type (negative control) mice had minimal tau pathology in the infused or contralateral hippocampus. Infusion of the $40 \%$ sucrose gradient fraction also showed substantial induction and spread of PG-5-positive tau pathology, whereas mice infused with the $10 \%$ sucrose gradient fraction were indistinguishable to wild-type lysate infused mice. Infusing a preparation of TgP3015 brainstem lysate that was normalized to have comparable DA9 levels (total tau) to the 10 and $40 \%$ sucrose gradient fractions did not show robust tau propagation. B, Quantification of PG-5-positive immunoreactivity in the infused hippocampus showed significantly higher levels of tau pathology in mice infused with TgP301S brainstem lysate compared with all other groups and a significant increase associated with the $40 \%$ sucrose gradient fraction when compared with wild-type and $10 \%$ sucrose groups. C, Analysis of the contralateral hippocampus showed a similar pattern albeit tau pathology milder than the infused hippocampus. Images shown in $\boldsymbol{A}$ are representative of the group mean. Scale bar, $500 \mu \mathrm{m}$. Graphs in $\boldsymbol{B}$ and $\boldsymbol{C}$ display group mean \pm SEM. Statistical analyses: one-way ANOVA and least significance difference post hoc test; ${ }^{*} p<0.05,{ }^{* *} p<0.01, \# p<0.01$ for all groupwise comparisons.

than those in the $40 \%$ fraction and seeded less well, in accordance with findings showing that long filaments are taken up less efficiently by cells than short filaments (Wu et al., 2013). This was also supported by the fact that the pellet present at the end of the centrifugation was only highly seeding-competent after sonication, which fragments filaments (data not shown).

Of significance was the finding that the $10 \%$ fraction failed to seed tau aggregation, despite containing the $400 \mathrm{kDa}$ oligomeric tau species with post-translational modifications, similar to those of the larger assemblies (i.e., AT8/nY29-positive). It is conceivable that this oligomeric species is a non-seed-competent precur- sor of larger fibrils, and could thus represent an intermediate but relatively stable aggregation step between monomeric and short fibrillar tau. This suggests that in the TgP301S mice tau aggregation may proceed via nucleated conformational conversion (oligomers form, which are then converted into a nucleus and amyloid fibrils through monomer addition) rather than nucleated polymerization (a high-energy nucleus forms, which grows into oligomers and amyloid fibrils through monomer addition) as in the latter, the $10 \%$ fraction would be expected to be seedcompetent. This mechanism of aggregation has been proposed for prions (Serio et al., 2000; Eisele et al., 2015). However, addi- 
tional studies will be required to examine this hypothesis and determine whether it is broadly applicable to tau aggregation in human tauopathy. Although we found no evidence of seedcompetent small (low- $n$ ) oligomeric tau assemblies in brain extracts from TgP301S mice, this outcome is in contrast with a recent report that a tau trimer was the smallest assembly that could seed intracellular tau aggregation (Mirbaha et al., 2015). However, this study used different methods (they examined the ability of a trimer to promote dimerization of tau repeats with the P301S mutation) and importantly did not examine tau seeding and propagation in vivo. In addition, although Mirbaha et al. (2015) reported that a tau trimer was capable of seeding intracellular tau aggregation, they observed more efficient seeding with larger assemblies, which is consistent with our findings.

Upon injection into the brains of presymptomatic TgP301S tau mice, using brainstem lysates from symptomatic mice as the control, the $10 \%$ sucrose gradient fraction containing oligomeric tau, failed to promote tau aggregation. By contrast, intrahippocampal injection of the $40 \%$ fraction containing short filamentous tau, led to a robust induction of tau pathology, which spread to the contralateral side and the mammillary nucleus. When normalized for the amount of total tau, the $40 \%$ fraction was more potent at seeding tau aggregation than the total brainstem lysate. Our findings imply that aggregation and the formation of a critical conformation associated with filament assembly, most likely a cross- $\beta$ structure based on comparisons with similar amyloidogenic proteins, is required for seeding tau aggregation in cell and animal models (Walker and Jucker, 2015).

Together, the findings from the characterization of the sucrose gradient fractions suggest that the formation of large tau assemblies ( $>10$ mers) is required for seeding tau aggregation in our cell model and in vivo assay. Specifically tau that was aggregated, AT8-, and nY29-positive with variant structures (ranging from ring-like to small fibrils of $179-250 \mathrm{~nm}$ ) were able to seed tau aggregation in our cell model and initiate trans-synaptic spreading in vivo. These results indicate that a diverse population of aggregates are capable of seeding tau pathology, with the most potent species being short fibrils. Larger fibrils and filaments $(>250 \mathrm{~nm})$ were less potent at seeding aggregation suggesting that there is an upper limit on the size of the assemblies that are able to efficiently seed tau aggregation. The short fibrils in the $\mathrm{TgP} 301 \mathrm{~S}$ brain extracts may be generated by filament fragmentation and/or secondary nucleation, consistent with findings for other amyloidogenic proteins (for review, see Prusiner, 2013; Walker and Jucker, 2015).

It remains to be determined whether the seed-competent tau species can also be neurotoxic. Neither the brainstem lysate, nor the 10 or $40 \%$ sucrose gradient fractions from the brains of symptomatic TgP301S tau mice induced nerve cell loss for up to 2.5 months after injection, despite the formation of widespread neurofibrillary pathology. It is conceivable that the propagation of inclusions, although necessary, is not sufficient for neurodegeneration. The tau species responsible for neurotoxicity may thus be different from those required for propagation (Clavaguera et al., 2009; Ahmed et al., 2014). Toxicity may require the generation of tau species by secondary nucleation after initial tau spreading and propagation together with longer incubation times and/or larger amounts of seed-competent tau to result in neurodegeneration. The present study shows that small fibrils are the major seedcompetent tau species in the TgP301S mouse model. In future, it will be important to determine whether similar species of aggregated tau underlie seeding, spreading, and neurodegeneration in Alzheimer's disease and other human tauopathies.

\section{References}

Ahmed Z, Cooper J, Murray TK, Garn K, McNaughton E, Clarke H, Parhizkar S, Ward MA, Cavallini A, Jackson S, Bose S, Clavaguera F, Tolnay M, Lavenir I, Goedert M, Hutton ML, O'Neill MJ (2014) A novel in vivo model of tau propagation with rapid and progressive neurofibrillary tangle pathology: the pattern of spread is determined by connectivity, not proximity. Acta Neuropathol 127:667-683. CrossRef Medline

Allen B, Ingram E, Takao M, Smith MJ, Jakes R, Virdee K, Yoshida H, Holzer M, Craxton M, Emson PC, Atzori C, Migheli A, Crowther RA, Ghetti B, Spillantini MG, Goedert M (2002) Abundant tau filaments and nonapoptotic neurodegeneration in transgenic mice expressing human P301S tau protein. J Neurosci 22:9340-9351. Medline

Berger Z, Roder H, Hanna A, Carlson A, Rangachari V, Yue M, Wszolek Z, Ashe K, Knight J, Dickson D, Andorfer C, Rosenberry TL, Lewis J, Hutton M, Janus C (2007) Accumulation of pathological tau species and memory loss in a conditional model of tauopathy. J Neurosci 27:3650-3662. CrossRef Medline

Braak H, Braak E (1991) Neuropathological stageing of Alzheimer-related changes. Acta Neuropathol 82:239-259. CrossRef Medline

Brettschneider J, Del Tredici K, Lee VM, Trojanowski JQ (2015) Spreading of pathology in neurodegenerative diseases: a focus on human studies. Nat Rev Neurosci 16:109-120. CrossRef Medline

Cavallini A, Brewerton S, Bell A, Sargent S, Glover S, Hardy C, Moore R, Calley J, Ramachandran D, Poidinger M, Karran E, Davies P, Hutton M, Szekeres P, Bose S (2013) An unbiased approach to identifying tau kinases that phosphorylate tau at sites associated with Alzheimer's disease. J Biol Chem 288:23331-23347. CrossRef Medline

Clavaguera F, Bolmont T, Crowther RA, Abramowski D, Frank S, Probst A, Fraser G, Stalder AK, Beibel M, Staufenbiel M, Jucker M, Goedert M, Tolnay M (2009) Transmission and spreading of tauopathy in transgenic mouse brain. Nat Cell Biol 11:909-913. CrossRef Medline

Clavaguera F, Lavenir I, Falcon B, Frank S, Goedert M, Tolnay M (2013a) "Prion-like" templated misfolding in tauopathies. Brain Pathol 23: 342-349. CrossRef Medline

Clavaguera F, Akatsu H, Fraser G, Crowther RA, Frank S, Hench J, Probst A, Winkler DT, Reichwald J, Staufenbiel M, Ghetti B, Goedert M, Tolnay M (2013b) Brain homogenates from human tauopathies induce tau inclusions in mouse brain. Proc Natl Acad Sci U S A 110:9535-9540. CrossRef Medline

de Calignon A, Polydoro M, Suárez-Calvet M, William C, Adamowicz DH, Kopeikina KJ, Pitstick R, Sahara N, Ashe KH, Carlson GA, Spires-Jones TL, Hyman BT (2012) Propagation of tau pathology in a model of early Alzheimer's disease. Neuron 73:685-697. CrossRef Medline

DeMattos RB, Rudel LL, Williams DL (2001) Biochemical analysis of cellderived apoE3 particles active in stimulating neurite outgrowth. J Lipid Res 42:976-987. Medline

Eisele YS, Monteiro C, Fearns C, Encalada SE, Wiseman RL, Powers ET, Kelly JW (2015) Targeting protein aggregation for the treatment of degenerative diseases. Nat Rev Drug Discov 14:759-780. CrossRef Medline

Falcon B, Cavallini A, Angers R, Glover S, Murray TK, Barnham L, Jackson S, O'Neill MJ, Isaacs AM, Hutton ML, Szekeres PG, Goedert M, Bose S (2015) Characterisation of native and synthetic tau species critical for tau seeding in a cell based model of tauopathy. J Biol Chem 290:1049-1065. CrossRef Medline

Frost B, Jacks RL, Diamond MI (2009) Propagation of tau misfolding from the outside to the inside of a cell. J Biol Chem 284:12845-12852. CrossRef Medline

Goedert M (2015) Alzheimer's and Parkinson's diseases: the prion concept in relation to assembled $\mathrm{A} \beta$, tau, and $\alpha$-synuclein. Science 349:1255555. CrossRef Medline

Goedert M, Spillantini MG, Jakes R, Rutherford D, Crowther RA (1989) Multiple isoforms of human microtubule-associated protein tau: sequences and localisation in neurofibrillary tanles of Alzheimer's disease. Neuron 3:519-526. CrossRef Medline

Greenberg SG, Davies P, Schein JD, Binder LI (1992) Hydrofluoric acidtreated tau PHF proteins display the same biochemical properties as normal tau. J Biol Chem 267:564-569. Medline

Guo JL, Lee VM (2011) Seeding of normal tau by pathological tau conform- 
ers drives pathogenesis of Alzheimer-like tangles. J Biol Chem 286: 15317-15331. CrossRef Medline

Holmes BB, DeVos SL, Kfoury N, Li M, Jacks R, Yanamandra K, Ouidja MO, Brodsky FM, Marasa J, Bagchi DP, Kotzbauer PT, Miller TM, PapyGarcia D, Diamond MI (2013) Heparan sulfate proteoglycans mediate internalization and propagation of specific proteopathic seeds. Proc Natl Acad Sci U S A 110:E3138-3147. CrossRef Medline

Iba M, Guo JL, McBride JD, Zhang B, Trojanowski JQ, Lee VM (2013) Synthetic tau fibrils mediate transmission of neurofibrillary tangles in a transgenic mouse model of Alzheimer's-like tauopathy. J Neurosci 33: 1024-1037. CrossRef Medline

Jicha GA, O’Donnell A, Weaver C, Angeletti R, Davies P (1999) Hierarchical phosphorylation of recombinant tau by the paired-helical filamentassociated protein kinase is dependent on cyclic AMP-dependent protein kinase. J Neurochem 72:214-224. CrossRef Medline

Kfoury N, Holmes BB, Jiang H, Holtzman DM, Diamond MI (2012) Transcellular propagation of tau aggregation by fibrillar species. J Biol Chem 287:19440-19451. CrossRef Medline

Liu L, Drouet V, Wu JW, Witter MP, Small SA, Clelland C, Duff K (2012) Trans-synaptic spread of tau pathology in vivo. PLoS One 7:e31302. CrossRef Medline

Maeda S, Sahara N, Saito Y, Murayama S, Ikai A, Takashima A (2006) Increased levels of granular tau oligomers: an early sign of brain aging and Alzheimer's disease. Neurosci Res 54:197-201. CrossRef Medline

Mirbaha H, Holmes BB, Sanders DW, Bieschke J, Diamond MI (2015) Tau trimers are the minimal propagation unit spontaneously internalized to seed intracellular aggregation. J Biol Chem 290:14893-14903. CrossRef Medline
Prusiner SB (2013) Biology and genetics of prions causing neurodegeneration. Annu Rev Genet 47:601-623. CrossRef Medline

Sanders DW, Kaufman SK, De Vos SL, Sharma AM, Mirbaha H, Li A, Barker SJ, Foley AC, Thorpe JR, Serpell LC, Miller TM, Grinberg LT, Seeley WW, Diamond MI (2014) Distinct tau prion strains propagate in cells and mice and define different tauopathies. Neuron 82:1271-1288. CrossRef Medline

Santa-Maria I, Varghese M, Ksiezak-Reding H, Dzhun A, Wang J, Pasinetti GM (2012) Paired helical filaments from Alzheimer disease brain induce intracellular accumulation of tau protein in aggresomes. J Biol Chem 287:20522-20533. CrossRef Medline

Serio TR, Cashikar AG, Kowal AS, Sawicki GJ, Moslehi JJ, Serpell L, Arnsdorf MF, Lindquist SL (2000) Nucleated conformational conversion and the replication of conformational information by a prion determinant. Science 289:1317-1321. CrossRef Medline

Spillantini MG, Goedert M (2013) Tau pathology and neurodegeneration. Lancet Neurol 12:609-622. CrossRef Medline

Tremblay MA, Acker CM, Davies P (2010) Tau phosphorylated at tyrosine 394 is found in Alzheimer's disease tangles and can be a product of the Abl-related kinase, Arg. J Alzheimers Dis 19:721-733. CrossRef Medline

Vincent I, Rosado M, Davies P (1996) Mitotic mechanisms in Alzheimer's disease? J Cell Biol 132:413-425. CrossRef Medline

Walker LC, Jucker M (2015) Neurodegenerative diseases: expanding the prion concept. Annu Rev Neurosci 38:87-103. CrossRef Medline

Wu JW, Herman M, Liu L, Simoes S, Acker CM, Figueroa H, Steinberg JI, Margittai M, Kayed R, Zurzolo C, Di Paolo G, Duff KE (2013) Small misfolded tau species are internalized via bulk endocytosis and anterogradely and retrogradely transported in neurons. J Biol Chem 288: 1856-1870. CrossRef Medline 